\title{
Necklace bisection with one cut less than needed
}

\author{
Gábor Simonyi* \\ Alfréd Rényi Institute of Mathematics \\ Hungarian Academy of Sciences \\ 1364 Budapest, POB 127, Hungary \\ simonyi@renyi.hu
}

Submitted: Jun 28, 2007; Accepted: May 26, 2008; Published: May 31, 2008

Mathematics Subject Classifications: 05A18, 05D99, 55M20

\begin{abstract}
A well-known theorem of Goldberg and West states that two thieves can always split a necklace containing an even number of beads from each of $k$ types fairly by at most $k$ cuts. We prove that if we can use at most $k-1$ cuts and fair splitting is not possible then the thieves still have the following option. Whatever way they specify two disjoint sets $D_{1}, D_{2}$ of the types of beads with $D_{1} \cup D_{2} \neq \emptyset$, it will always be possible to cut the necklace (with $k-1$ cuts) so that the first thief gets more of those types of beads that are in $D_{1}$ and the second gets more of those in $D_{2}$, while the rest is divided equally. The proof combines the simple proof given by Alon and West to the original statement with a variant of the Borsuk-Ulam theorem due to Tucker and Bacon.
\end{abstract}

\section{Introduction}

The Borsuk-Ulam theorem is successfully applied in combinatorics ever since the appearance of Lovász's [8] celebrated proof of Kneser's conjecture. This result and many of the developments it triggered are presented in detail in Matoušek's excellent monograph [9]. These include several generalizations of the Lovász-Kneser theorem that appeared over the years. Some further generalizations that are based on generalizations of the BorsukUlam theorem due to Ky Fan [4] and due to Tucker [15] and Bacon [3] appeared more recently in $[10,12,13]$, cf. also [5].

Besides coloring Kneser-like graphs, another combinatorial setting where the BorsukUlam theorem is successfully applied is the area of necklace splitting results, cf. [1, 2, 6]. The main goal of this note is to show what necklace splitting type statement can be deduced by applying the above mentioned theorem of Tucker and Bacon.

\footnotetext{
${ }^{*}$ Research partially supported by the Hungarian Foundation for Scientific Research Grant (OTKA) Nos. T046376, AT048826, NK62321
} 


\section{$2 \quad$ Necklace bisections}

The necklace splitting problem is traditionally introduced (cf. [1, 2]) by referring to two mathematically oriented thieves who stole a necklace with $2 a_{i}$ beads of type $i$ for $i=1, \ldots, k$ and want to divide it among themselves with a minimum number of cuts achieving the goal that both of them get exactly half of the beads of each type. If all beads of type $i$ are to the left of all beads of type $i+1$ for every $i=1, \ldots, k-1$ then one can easily see the necessity of $k$ cuts. It is shown in [6] and reproved in a simpler way in [2] that this lower bound is sharp, i.e., $k$ cuts always suffice. A generalization to many thieves is proven in [1], but here we are concerned only with the case of two thieves.

The simple proof of Alon and West [2] proves the following more general statement, called the Hobby-Rice theorem [7].

Theorem 1 ([7], cf. also [2]) Given $g_{1}, \ldots, g_{k}:[0,1] \rightarrow \mathbb{R}$ continously integrable functions there always exist $0=z_{0} \leq z_{1} \leq \ldots \leq z_{k} \leq z_{k+1}=1$ and $\delta_{1}, \ldots, \delta_{k+1} \in\{+1,-1\}$ such that $\Sigma_{i=1}^{k+1} \delta_{i} \int_{z_{i-1}}^{z_{i}} g_{j}=0$ for all $1 \leq j \leq k$.

What we prove here is the following variant. (As we will see from the Remark ending this section, it actually implies Theorem 1.)

Theorem 2 Let $g_{1}, \ldots, g_{k}:[0,1] \rightarrow \mathbb{R}$ be continously integrable functions. Either there exist $0=z_{0} \leq z_{1} \leq \ldots \leq z_{k-1} \leq z_{k}=1$ and $\delta_{1}, \ldots, \delta_{k} \in\{+1,-1\}$ such that $\Sigma_{i=1}^{k} \delta_{i} \int_{z_{i-1}}^{z_{i}} g_{j}=0$ for all $1 \leq j \leq k$ or for every $\beta_{1}, \ldots, \beta_{k} \in\{+,-, 0\}$ that are not all 0 , there exist $0=z_{0} \leq z_{1} \leq \ldots \leq z_{k-1} \leq z_{k}=1$ and $\delta_{1}, \ldots, \delta_{k} \in\{+1,-1\}$ such that the sign of $\sum_{i=1}^{k} \delta_{i} \int_{z_{i-1}}^{z_{i}} g_{j}$ equals $\beta_{j}$ for every $1 \leq j \leq k$.

In the language of necklaces and thieves this has the following consequence. We use the phrase necklace bisection with the same meaning as in [2], i.e., it is a collection of cuts together with a bipartition of the segments thus created. We also follow [2] by calling the types of beads colors.

Corollary 3 Let us be given a necklace containing an even number of beads in each of $k$ colors. If there is no bisection using at most $k-1$ cuts and resulting in a fair bipartition of the beads, i.e., one that puts exactly half of the beads of each color into both partition classes, then for any possible prescribed disjoint subsets $D_{1}, D_{2}$, not both empty, of the color set $\{1, \ldots, k\}$ there exists a bisection of the necklace using at most $k-1$ cuts and resulting in a bipartition of the beads that puts more beads of color $i$ into partition class $j$ if and only if $i \in D_{j}$.

That is, if we are short of just one cut and it is indeed not possible to make a fair division of the beads then the two thieves can agree on two arbitrary disjoint nonempty (at least not both empty) subsets of the colors that mark the types of beads one and the other gets more of. 
Corollary 3 follows from Theorem 2 the same way as the original necklace bisection theorem follows from Theorem 1 in [2]. We only say briefly, that the functions $g_{i}$ are viewed as the mass functions for the beads of color $i$ on the unit interval representing the necklace. (Some rounding problems may occur that are not very difficult to resolve.) For the details we refer to [2], cf. also [9] for a thorough explanation. Theorem 2 is proven in the next section.

Remark. (This remark is due to Gábor Tardos [14].) It is worth noting that Corollary 3 is a direct generalization of the original necklace bisection theorem. Indeed, if we have beads of $k$ colors and we can use $k$ cuts then we can apply Corollary 3 by adding a fictitious extra color and choosing $D_{1}$ to contain only this new color while $D_{2}$ to be empty. It follows that with $k$ cuts we can fairly divide the beads of the existing $k$ colors. Clearly, the same argument shows that Theorem 2 generalizes Theorem 1.

\section{The proof}

For proving Theorem 2 we just have to translate (routinly) the proof of Alon and West [2] that uses a classical form of the Borsuk-Ulam theorem into one using another classical form, the so-called Lyusternik-Shnirel'man version (cf. [9]) and then substitute the latter by the theorem of Tucker and Bacon. To this end we first state the Tucker-Bacon theorem. In [13] a little more general form of this theorem is given than the one appearing in [3]. Here we give a still little more general form which would not have been relevant in the applications for graph coloring in [13], but has some relevance in the present context.

Tucker-Bacon theorem. Let $A_{1}, \ldots, A_{h+1}$ be open subsets of the $h$-dimensional sphere $\mathbb{S}^{h}$ satisfying that $\forall i A_{i} \cap\left(-A_{i}\right)=\emptyset$ and $\bigcup_{i=1}^{h+1}\left(A_{i} \cup\left(-A_{i}\right)\right)=\mathbb{S}^{h}$. Then for any pair of disjoint sets $B, C \subseteq\{1, \ldots, h+1\}$ for which $B \cup C \neq \emptyset$ there exists a point $\boldsymbol{x} \in \mathbb{S}^{h}$ such that $\boldsymbol{x} \in A_{i}$ iff $i \in B$ and $-\boldsymbol{x} \in A_{i}$ iff $i \in C$.

As this statement is a bit more general than its predecessors mentioned above, we say a few words about the proof. (The difference compared to the form given in [13] is that here we do not insist on $B \cup C=\{1, \ldots, h+1\}$.) The argument uses a simple idea that also appears in Zhu's [16] presentation of some results of [10], [12] and it was also observed by Carsten Schultz [11]. The basic method is also similar to those used in [12, 13].

Sketch of proof of the above form of the Tucker-Bacon theorem. Let the open sets $A_{i} \subseteq \mathbb{S}^{h}$ be given as in the statement. One can define an antipodal map from $\mathbb{S}^{h}$ to the surface of the $(h+1)$-dimensional crosspolytope. The latter is the convex hull of its vertices $\pm \boldsymbol{v}_{\boldsymbol{i}}$, where $\boldsymbol{v}_{\boldsymbol{i}}=(0, \ldots, 0,1,0, \ldots, 0)$ is the unit vector in the direction of the $i^{\text {th }}$ coordinate axis. We consider these $2(h+1)$ vertices to be in one-to-one correspondence with the sets $\pm A_{i}$ so that $\boldsymbol{v}_{\boldsymbol{j}}$ corresponds to $A_{j}$ and $-\boldsymbol{v}_{\boldsymbol{j}}$ corresponds to $-A_{j}$. The vertices of the unique minimal face that contains the image of an $\boldsymbol{x} \in \mathbb{S}^{h}$ will be exactly those vertices that are corresponding to the sets $\pm A_{i}$ that contain $\boldsymbol{x}$. A possible such map is $f(\boldsymbol{x})=\frac{1}{D(\boldsymbol{x})+D(-\boldsymbol{x})} \sum_{i=1}^{h+1}\left(d(\boldsymbol{x}, i) \boldsymbol{v}_{\boldsymbol{i}}+d(-\boldsymbol{x}, i)\left(-\boldsymbol{v}_{\boldsymbol{i}}\right)\right)$, where $d(\boldsymbol{y}, i)$ denotes the distance of $\boldsymbol{y}$ from the (closest point of the) set $\mathbb{S}^{h} \backslash A_{i}$ and $D(\boldsymbol{y})=\sum_{i=1}^{h+1} d(\boldsymbol{y}, i)$. Note that 
$d(\boldsymbol{y}, i)>0$ is equivalent to $\boldsymbol{y} \in A_{i}$ and thus the condition $\bigcup_{i=1}^{h+1}\left(A_{i} \cup\left(-A_{i}\right)\right)=\mathbb{S}^{h}$ implies $D(\boldsymbol{y})+D(-\boldsymbol{y})>0$ for all $\boldsymbol{y} \in \mathbb{S}^{h}$, while $A_{i} \cap\left(-A_{i}\right)=\emptyset$ implies that $f(\boldsymbol{x})$ is indeed on the surface of the crosspolytope.

As the given map is a continuous antipodal map it should be onto. If it were not, then, by homotopy equivalence of the surface of the crosspolytope to $\mathbb{S}^{h}$, it would provide an antipodal map from $S^{h}$ to a lower dimensional sphere (that we get from the surface of the crosspolytope by deleting two of its antipodal points) and such a map cannot exist according to a well-known form of the Borsuk-Ulam theorem, see, e.g., [9].

Thus the inverse image of a point of the crosspolytope which lies inside its face with set of vertices $\left\{\boldsymbol{v}_{\boldsymbol{i}}: i \in B\right\} \cup\left\{-\boldsymbol{v}_{\boldsymbol{j}}: j \in C\right\}$ satisfies the statement.

Proof of Theorem 2. Consider each point $\boldsymbol{x}=\left(x_{1}, \ldots, x_{k}\right) \in \mathbb{S}^{k-1}$ to be an encoding of the numbers $z_{i}(\boldsymbol{x})$ and $\delta_{i}(\boldsymbol{x})$ as in [2], cf. also [9], i.e., such that $z_{i}(\boldsymbol{x})=\sum_{j=1}^{i} x_{j}^{2}$ for $i=1, \ldots, k$ and $\delta_{i}(\boldsymbol{x})$ having the same sign as $x_{i}$. Define sets $A_{1}, \ldots, A_{k} \in S^{k-1}$ as follows. For an $\boldsymbol{x} \in S^{k-1}$ we have $\boldsymbol{x} \in A_{j}$ if and only if $\sum_{i=1}^{k} \delta_{i}(\boldsymbol{x}) \int_{z_{i-1}(\boldsymbol{x})}^{z_{i}(\boldsymbol{x})} g_{j}>0$. It is easy to see that these sets $A_{j}$ are open and $A_{j} \cap\left(-A_{j}\right)=\emptyset$ holds for each $j$. If there is some $\boldsymbol{x} \in \mathbb{S}^{k-1}$ for which $\sum_{i=1}^{k} \delta_{i}(\boldsymbol{x}) \int_{z_{i-1}(\boldsymbol{x})}^{z_{i}(\boldsymbol{x})} g_{j}=0$ for all $j$ then we are done. If there is

no such $\boldsymbol{x}$ then $\bigcup_{i=1}^{k}\left(A_{i} \cup\left(-A_{i}\right)\right)=\mathbb{S}^{k-1}$ and thus the conditions of the Tucker-Bacon theorem are satisfied. Apply it to $B:=\left\{i: \beta_{i}=+\right\}$ and $C:=\left\{i: \beta_{i}=-\right\}$. The Tucker-Bacon theorem then outputs an $\boldsymbol{x} \in S^{k-1}$ that defines appropriate numbers $z_{i}(\boldsymbol{x})$ and $\delta_{i}(\boldsymbol{x})$ which will have the required properties by the definition of the sets $A_{i}$.

Acknowledgement. Thanks are due to Gábor Tardos for his several useful comments.

\section{References}

[1] N. Alon, Splitting necklaces, Adv. in Math., 63 (1987), no. 3, 247-253.

[2] N. Alon, D. B. West, The Borsuk-Ulam theorem and bisection of necklaces, Proc. Amer. Math. Soc., 98 (1986), no. 4, 623-628.

[3] P. Bacon, Equivalent formulations of the Borsuk-Ulam theorem, Canad. J. Math., 18 (1966), 492-502.

[4] K. Fan, A generalization of Tucker's combinatorial lemma with topological applications, Annals of Mathematics, 56 (1952), no. 2, 431-437.

[5] K. Fan, Evenly distributed subsets of $\mathbb{S}^{n}$ and a combinatorial application, Pacific J. Math., 98 (1982), no. 2, 323-325.

[6] C. H. Goldberg, D. B. West, Bisection of circle colorings, SIAM J. Algebraic Discrete Methods, 6 (1985), no. 1, 93-106.

[7] C. R. Hobby, J. R. Rice, A moment problem in $L_{1}$ approximation, Proc. Amer. Math. Soc., 16 (1965), 665-670.

[8] L. Lovász, Kneser's conjecture, chromatic number, and homotopy, J. Combin. Theory Ser. A, 25 (1978), no. 3, 319-324. 
[9] J. Matoušek, Using the Borsuk-Ulam Theorem. Lectures on Topological Methods in Combinatorics and Geometry, Universitext, Springer-Verlag, Heidelberg, 2003.

[10] F. Meunier, A topological lower bound for the circular chromatic number of Schrijver graphs, J. Graph Theory, 49 (2005), 257-261.

[11] C. Schultz, private communication (Oberwolfach, 2007).

[12] G. Simonyi, G. Tardos, Local chromatic number, Ky Fan's theorem, and circular colorings, Combinatorica, 26 (2006), 587-626. arXiv:math.CO/0407075.

[13] G. Simonyi, G. Tardos, Colorful subgraphs of Kneser-like graphs, European J. Combin. 28 (2007), 2188-2200. arxiv:math.CO/0512019.

[14] G. Tardos, private communication.

[15] A. W. Tucker, Some topological properties of disk and sphere, Proc. First Canadian Math. Congress, Montreal, 1945, University of Toronto Press, Toronto, 1946, 285309.

[16] X. Zhu, Recent developments in circular colouring of graphs, in: Topics in Discrete Mathematics, Algorithms Combin., 26, Springer-Verlag, Berlin, 2006, 497-550. 